\title{
Effects of Novel Hydantoin Derivatives with Aldose Reductase Inhibiting Activity on Galactose-Induced Cataract in Rats
}

\author{
Katsuaki KATO, Kazuo NAKAYAMA, Masahiko OHTA, Nobuya MURAKAMI, \\ Kimihiro MURAKAMI, Masahiro MIZOTA, Ichitomo MIWA ${ }^{1}$ and Jun OKUDA ${ }^{1}$ \\ Department of Pharmacology, Fuji Central Research Laboratory, \\ Mochida Pharmaceutical Co., Ltd., 1-1-1 Kamiya, Kita-ku. Tokyo 115, Japan \\ ${ }^{1}$ Department of Clinical Biochemistry. Faculty of Pharmacy, \\ Meijo University. Tempaku-cho. Tempaku-ku, Nagoya 468, Japan
}

Accepted August 21, 1990

\begin{abstract}
Effects of novel aldose reductase inhibitors, M16209 (1-13-bromobenzo[b]furan-2-y!sulfonyl) hydantoin) and M16287 (1-(3-chlorobenzo[b]furan-2ylsulfonyl) hydantoin), on galactose-induced cataract formation in rats were investigated. Rats fed a 30\% galactose diet developed lenticular opacity in the peripheral region by the 6 th day of galactose feeding and showed gradual progression of opacity from the equator to the center of lenses. Histological study on the 15th day showed apparent lens fiber swelling and vacuolation predominantly in the equatorial and anterior cortical regions. Biochemical changes such as accumulation of galactitol, depletion of myo-inositol and decrease in glutathione (GSH) content in lenses preceded the appearance of opacity. Remarkable increase in NADPH content and decrease in $\mathrm{NADP}^{+}$content, in addition to elevation of the ratio of $\mathrm{Na}^{+} / \mathrm{K}^{+}$, in lenses were also observed on the 15th day. Both M16209 and M16287 (10, 30 and $100 \mathrm{mg} / \mathrm{kg} /$ day, p.o.) dose-dependently ameliorated these morphological and biochemical changes except that restoration of myo-inositol content was incomplete. These results indicate that M16209 and M16287 can prevent galactose-induced cataract formation through amelioration of metabolic disorders and thus have high potential for clinical use in the treatment of some diabetic complications.
\end{abstract}

Since van Heyningen first reported the formation of polyols in the lens of the rat with sugar cataract (1), the osmotic theory has been promulgated by Kinoshita (2). The latter investigator suggested that the intracellular accumulation of polyol initiated by aldose reductase leads to an influx of water that results in permeability changes and eventually leads to a loss of cellular integrity. Therefore, the idea of using aldose reductase inhibitors for prevention of diabetic complications has been extensively examined over the past decade $(3,4)$. In recent years, it has been suggested that a decrease in myo-inositol content is involved in the pathogenesis of diabetic complications, particularly of diabetic neuropathy $(5,6)$. Altered myo-inositol metabolism and its coupling with the polyol pathway. however, has not been studied so extensively in terms of the pathogenesis of diabetic cataract, unlike that of diabetic neuropathy. On the other hand, much attention has been focused on the decreased antioxidant levels, particularly on the loss of glutathione (GSH). with respect to cataractogenesis in the aging lens (7). It was also suggested that aldose reductase inhibitors could restore the GSH level in lens with diabetic cataract and that normalization of the redox state of $\mathrm{NADP}^{+} /$ NADPH was implicated in the mechanism (8).

In the present study, therefore. we intended to examine the effects of M16209 and M16287, newly synthesized aldose reductase inhibitors, on galactose-induced cataractogenesis in rats and to investigate the biochemical mechanism substantially involved in the 
prevention of diabetic cataract by aldose reductase inhibitors.

\section{Materials and Methods}

Assay of aldose reductase inhibiting activity: Rat and bovine lens aldose reductases (RLAR and $B L A R$ ) were partially purified by 40-75\% ammonium sulfate fractionation of lens homogenate. Assay of aldose reductase was performed according to the procedure of Inagaki et al. (9) using Dt-glyceraldehyde as the substrate. The effect of an inhibitor on the enzyme activity was determined addition of $10 \mu$ of inhibitor solution in dimethyl sulfoxide (DMSO) at the desired concentrations. Inhibition rate (\%) was calculated regarding the enzyme activity with DMSO alone as $100 \%$. The concentration of an inhibitor required to produce $50 \%$ inhibition (IC50) was determined by plotting inhibition rate against log concentration of inhibitor.

Induction of galactosemic rats and administration of drugs: Male Sprague-Dawley rats (Japan SLC. Hamamatsu) weighing 90$120 \mathrm{~g}$ were fed a $30 \%$ galactose diet, prepared by mixing galactose with commercial powder chow, for 14 days at the longest. The rats were orally administered at around $10 \mathrm{a} . \mathrm{m}$. with a suspension of M16209 or M16287 (see Fig. 1 for chemical structures) in $5 \mathrm{~g} / \mathrm{dl}$ gum arabic solution from the first day of galactose feeding. The dosages of the compounds were 10 .

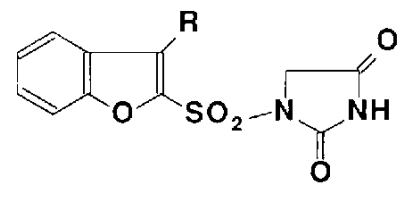

$$
\begin{aligned}
& M 16209: R=B r \\
& M 16287: R=C l
\end{aligned}
$$

Fig. 1. Chemical structures of M16209 and M16287.
30 and $100 \mathrm{mg} / \mathrm{kg} /$ day. Galactose-fed, untreated rats were administered vehicle alone. Non-galactosemic rats fed with ordinary chow were used as normal rats.

Examination of cataract formation: Morphological investigation was performed using a slit-lamp (Kowa SC-6. Nagoya) on the 2nd, 3 rd, 4th, 6th and 15th day of galactose feeding. Progression of the cataract was graded according to the method of Sippel (10) (see Table 1 for grading criteria). A lens reaching grade 1 was regarded to have a cataract. The rats were sacrificed by decapitation just after the final observation, and the eyes of rats were removed and fixed in 15\% formalin for a few days. Following dehydration in ethanol, they were embedded in paraffin, cut into sections and stained with toluidine blue for light microscopic examinations. Swollen, liquefied or vacuolated area of each preparation was measured using a computer-aided image analyzer (Nikon, Cosmo Zone ${ }^{(i)}$, Tokyo).

Measurement of biochemical changes in lenses: In order to investigate the time course of biochemical changes in lenses, separate groups of rats fed with galactose for $1,2,3,5$ and 14 days were treated in the same manner as described above. Lenses were removed and homogenized in distilled water. Homogenized samples were stored at $-40^{\circ} \mathrm{C}$ until use. Galactitol and myo-inositol contents were determined using high-performance luquid chromatography (11). GSH content was determined by a fluorophotometric method using o-phthalaldehyde (12). NADPH and $\mathrm{NADP}^{+}$were determined by the enzymatic cycling method (13), and $\mathrm{Na}^{+}$and $\mathrm{K}^{+}$contents were determined by a flamephotometric method (14) using an electrolytes autoanalyzer (Hitachi, model 710, Tokyo).

Statistical analysis: Data except for those of the slit-lamp analysis were expressed as the mean \pm S.E. Significance of the difference between groups was calculated by Student's

Table 1. Grading criteria of galactose cataract

Grade 1 Apparent opacification is observed in the peripheral region of the lens

Grade 2 Opacification is expanded into the central region up 10 1/4 of radius

Grade 3 Opacification up to $1 / 3$ of radius

Grade 4 Opacification up to $1 / 2$ of radius 
t-test.

\section{Results}

Inhibitory effects on partially purified aldose reductase: The concentrations of $\mathrm{M}$ 16209 and M16287 necessary for $50 \%$ inhibition of enzyme activity (IC50) were 0.12 and $0.08 \mu \mathrm{M}$, respectively, for partially purified RLAR. The IC50 values of M16209 and M16287 for partially purified BLAR were 0.24 and $0.25 \mu \mathrm{M}$, respectively.

Effects on cataract formation: Changes in incidence of cataract and mean grade of cataract formation by slit-lamp analysis are shown in Fig. 2A and Fig. 2B, respectively. Typical slit-lamp images of the lenses in normal rats and galactose-fed, untreated rats on the 6th and 15th days are shown in Fig. 3. All the rats in the untreated group developed

(A)

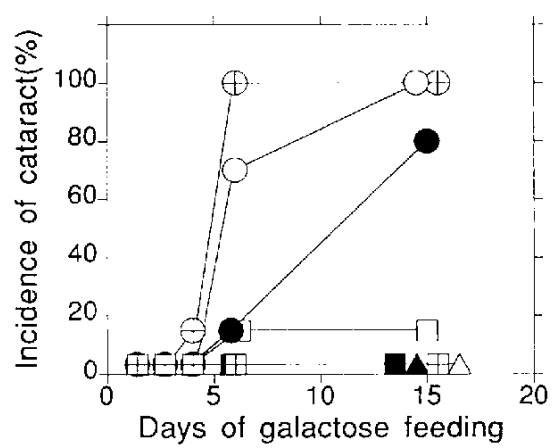

(B)

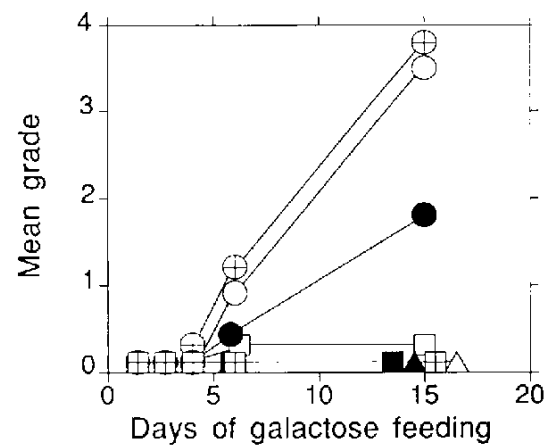

Fig. 2. Changes in incidence of cataract (A) and in mean grade (B) by slit-lamp analysis. Galactosefed, untreated $(\Theta)$; galactose $+M 16209: 10 \mathrm{mg} / \mathrm{kg}$ (O). $30 \mathrm{mg} / \mathrm{kg}(\square) .100 \mathrm{mg} / \mathrm{kg}(\Lambda):$ galactose + M16287: $10 \mathrm{mg} / \mathrm{kg}$ (O). $30 \mathrm{mg} / \mathrm{kg}$ (田). $100 \mathrm{mg} /$ $\mathrm{kg}(\mathbf{\Delta})$; and normal (T) group. lenticular opacity of grade 1 by the 6th day of galactose feeding, and the mean grade of the lenses reached about 4 on the 15th day. M16209 and M16287 prevented cataract formation in a dose-dependent manner. Nearly complete and complete prevention was observed at $30 \mathrm{mg} / \mathrm{kg} /$ day of $M 16209$ and $M 16287$, respectively. Typical images by light microscopy of the preparations from

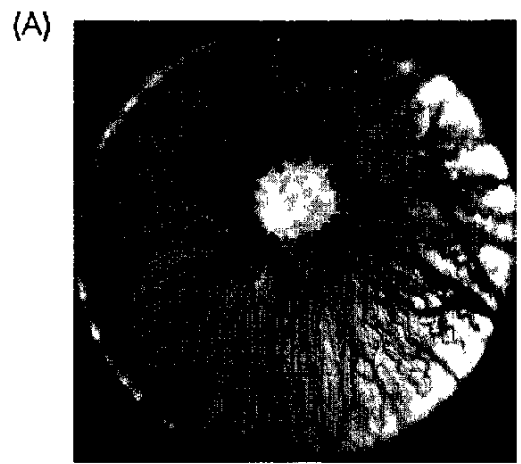

(B)

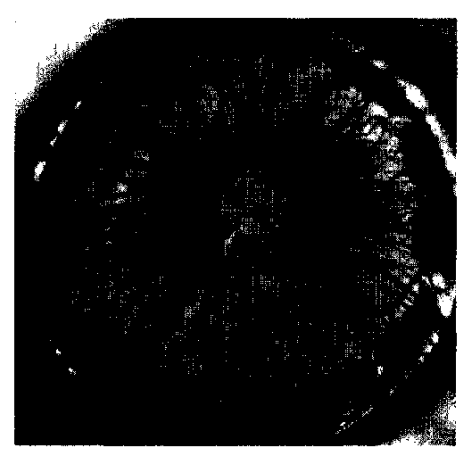

(C)

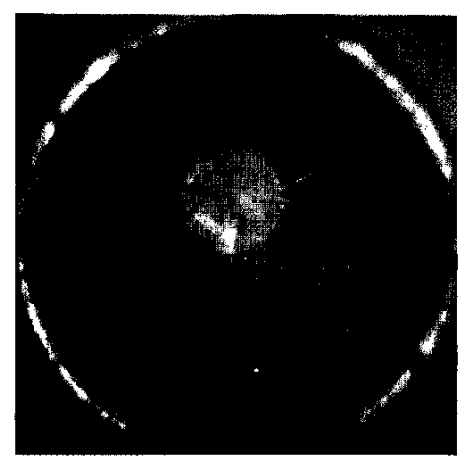

Fig. 3. Typical slit-lamp images of lenses in norma! rats $(A)$ and galactose-fed, untreated rats on the 6 th day (B) and the 15th day (C) of feeding. Apparent opacification in the peripheral region of the lens (grade 1) was observed in (B). Opacification up to $1 / 2$ of the radius (grade 4 ) was seen in (C). 
normal, untreated, M16209 and M16287 (30 $\mathrm{mg} / \mathrm{kg} /$ day) groups are shown in Fig. 4 . Fiber cell swelling and liquefaction or vacuo- lation were observed predominantly in the equatorial and anterior cortical regions in the untreated group. These histological abnor-

\section{Equatorial region}

(A)

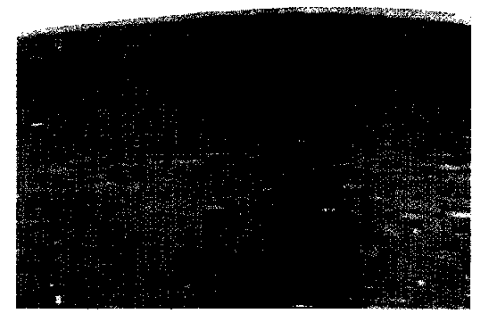

(B)

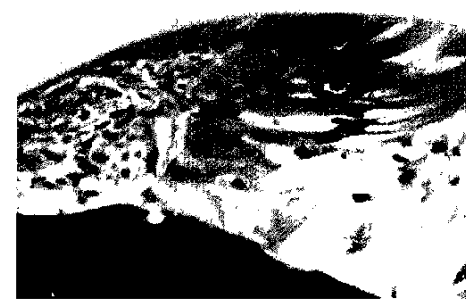

(C)

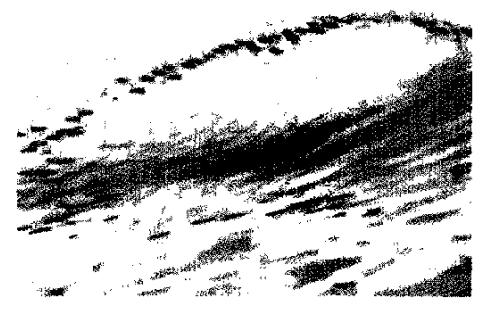

(D)

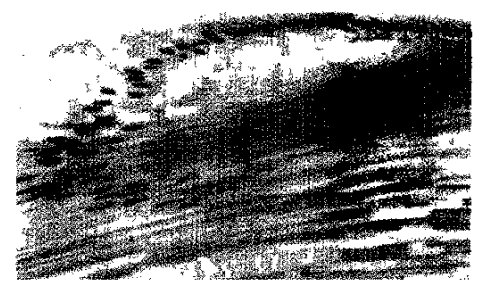

\section{Anterior region}
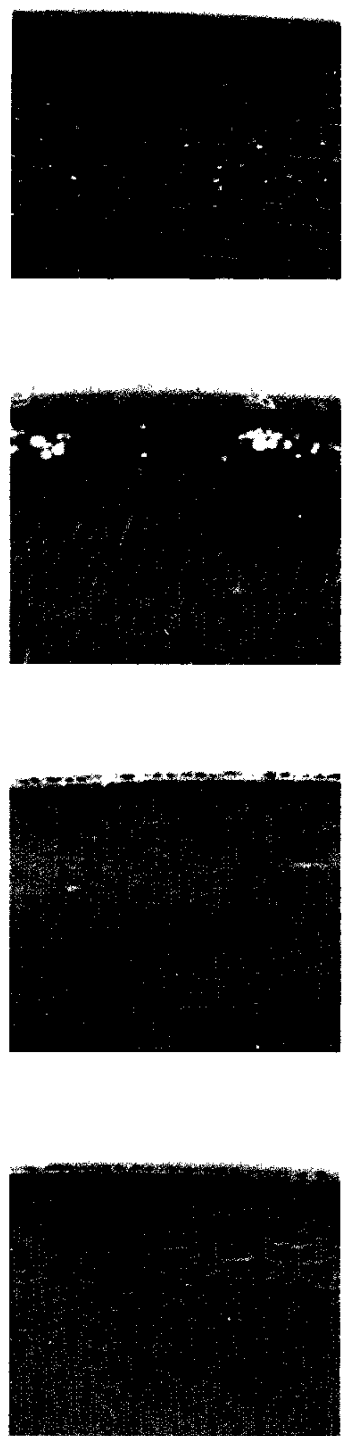

Fig. 4. Typical images of thin sections of lenses by light microscopy $(\times 50)$. Lenses were removed on the 15 th day of galactose feeding. Normal rats $(A)$ : no alteration was observed. Galactose-fed, untreated rats (B): large liquefied area, remarkable swelling of lens fiber and epithelial cells, abnormal epithelial population and disorganized bow region were observed in the equatorial region (left). and small liquefied area and lens fiber swelling were observed in the anterior region (right). Galactose-fed. M16209 (30 mg/kg/day)-treated rats (C) and M16287 (30 mg/kg/day)-treated rats (D): Compared with untreated rats, such alterations were remarkably suppressed. 
malities were ameliorated by the treatment with M16209 and M16287. Swollen, liquefied or vacuolated areas calculated by the image analyzer are shown in Table 2. M16209 and M16287 at a dose of $30 \mathrm{mg} / \mathrm{kg} /$ day significantly, though not completely, reduced the area of the affected region.

Effects on polyol content: Lens galactitol and myo-inositol contents in untreated, treated and normal groups are summarized in Table 3 . In the untreated group, there was a considerable accumulation of galactitol on the 2 nd day of galactose feeding, and the galactitol content reached its plateau on the 4 th day and remained at the same level until the 15th day. M16209 and M16287 dosedependently prevented the accumulation of galactitol. At the dose of $10 \mathrm{mg} / \mathrm{kg} / \mathrm{day}$, significant prevention was observed until the 3rd day (M16209) or the 4th day (M16287). At the doses of 30 and $100 \mathrm{mg} / \mathrm{kg} /$ day, there were significant differences between the untreated and treated groups throughout the observation period (M16209 and M16287). On the contrary, myo-inositol content rapidly decreased by galactose feeding and fell to an undetectable level in the untreated group on the 3rd day. M16209 and M16287 were able to delay myo-inositol depletion in a dose-dependent manner, but the myoinositol content fell to undetectable levels on the 15 th day even at the highest dose.

Effects on glutathione content: Lens GSH contents in the normal, untreated and treated groups are summarized in Table 4 . In the untreated group, there was a slight decrease in GSH content on the 2 nd day of galactose feeding followed by marked decrease on and after the 3rd day. M16209 and M16287 effectively prevented the decrease in GSH content.

Effects on NADPH and NADP ${ }^{+}$contents: $\mathrm{NADPH}$ and $\mathrm{NADP}^{+}$contents in the lenses are summarized in Table 5. The NADPH content in the untreated group was twice as much as that in the normal group. whereas the $\mathrm{NADP}^{+}$content in the former group was one tenth as much as that in the latter group. M16209 and M16287 dose-dependently prevented both the increase in NADPH and the decrease in $\mathrm{NADP}^{+}$

Effects on $\mathrm{Na}^{+}$and $\mathrm{K}^{+}$contents: $\mathrm{Na}^{+}$and $\mathrm{K}^{+}$contents in the lenses and the ratio of $\mathrm{Na}^{+} / \mathrm{K}^{+}$are summarized in Table 6 . The $\mathrm{Na}^{+}$ content in the untreated group was 1.6 times as much as that in the normal group; and on the other hand, the $\mathrm{K}^{+}$content in the untreated group was less than that in the normal group. The ratio of $\mathrm{Na}^{+} / \mathrm{K}^{+}$was clearly different between the untreated and normal groups. M16209 and M16287 normalized lens $\mathrm{Na}^{+}$ content, but not $\mathrm{K}^{+}$content, at doses of 30 and $100 \mathrm{mg} / \mathrm{kg} /$ day. The two drugs at the same doses consequently lowered the ratio of $\mathrm{Na}^{+} / \mathrm{K}^{+}$to the close-to-normal level.

\section{Discussion}

Gross observations of eyes using a slitlamp revealed that a grade 1 cataract began to develop on the 4th day of galactose feeding in the lenses of untreated rats and that the cataract progressed during the course of galactose feeding. On the other hand. changes in biochemical parameters such as lens polyol and glutathione contents were observed from earlier stages of galactose

Table 2. Effects of M16209 and M16287 on swollen, liquefied or vacuolated area (affected area) in the cross sections of rat lenses removed on the 15 th day of $30 \%$ galactose feeding

\begin{tabular}{lll}
\hline Group & $\mathrm{n}$ & Affected area $(\%)$ \\
\hline Galactose-fed & & \\
untreated & 5 & $22.3 \pm 1.9$ \\
+ M16209 $30 \mathrm{mg} / \mathrm{kg}$ & 5 & $14.5 \pm 2.1^{*}$ \\
+ M16287 $30 \mathrm{mg} / \mathrm{kg}$ & 5 & $12.5 \pm 1.4^{* *}$ \\
\hline
\end{tabular}

Affected area was limited in the peripheral region of the cross sections of lenses, and it could be easily distinguished from intact region by the intensity of toluidine blue staining at low magnification $(\times 20)$. The total area and intact area in the central region were measured by an image analyzer, and the difference between the former and the latter was regarcied as the affected area. The percentages of affected area (affected area/total area $\times 100)$ are shown. ${ }^{*}(P<0.05),{ }^{*}(P<0.01)$ : vs. untreated. 
K. Kato et al.

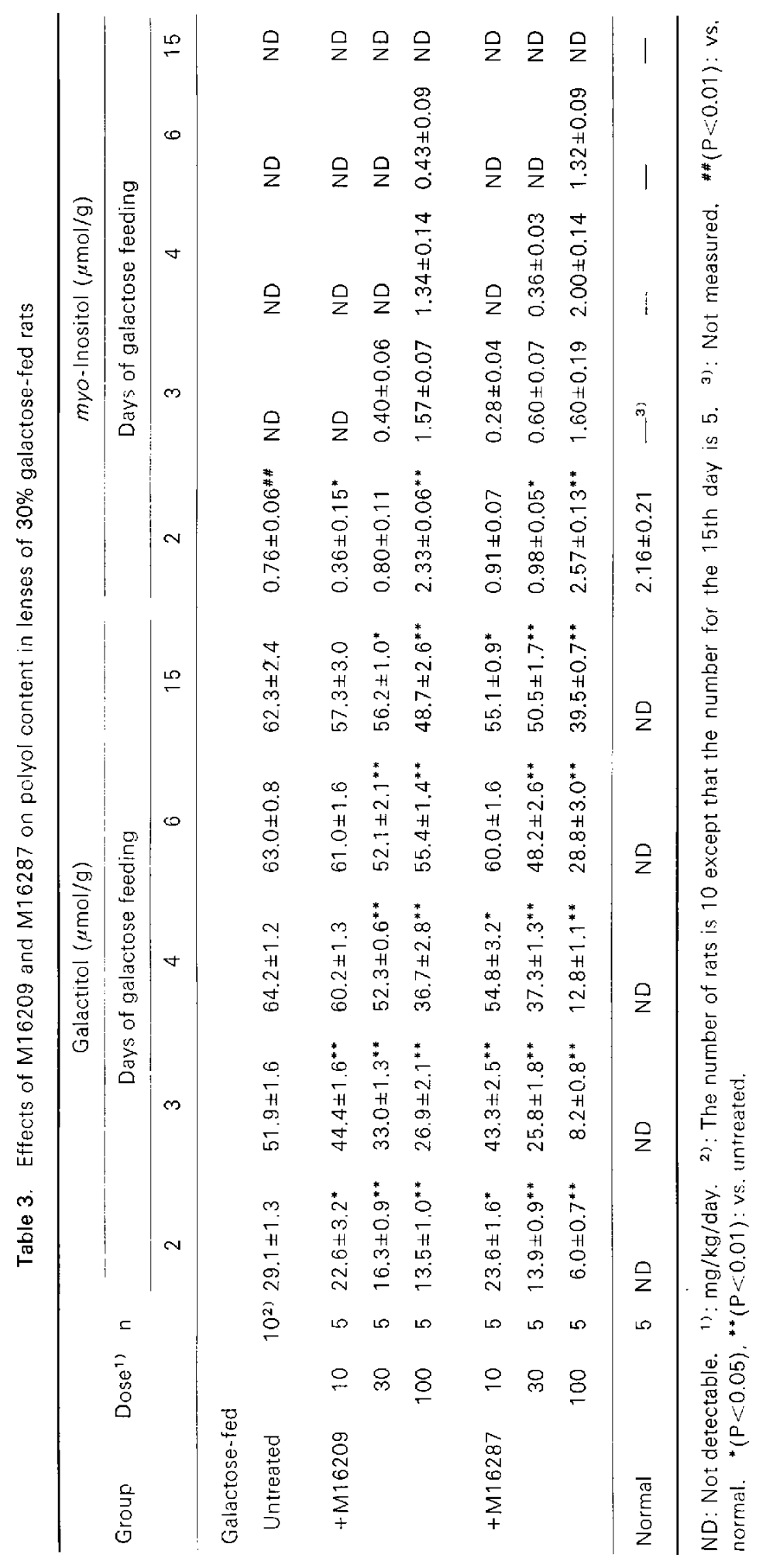




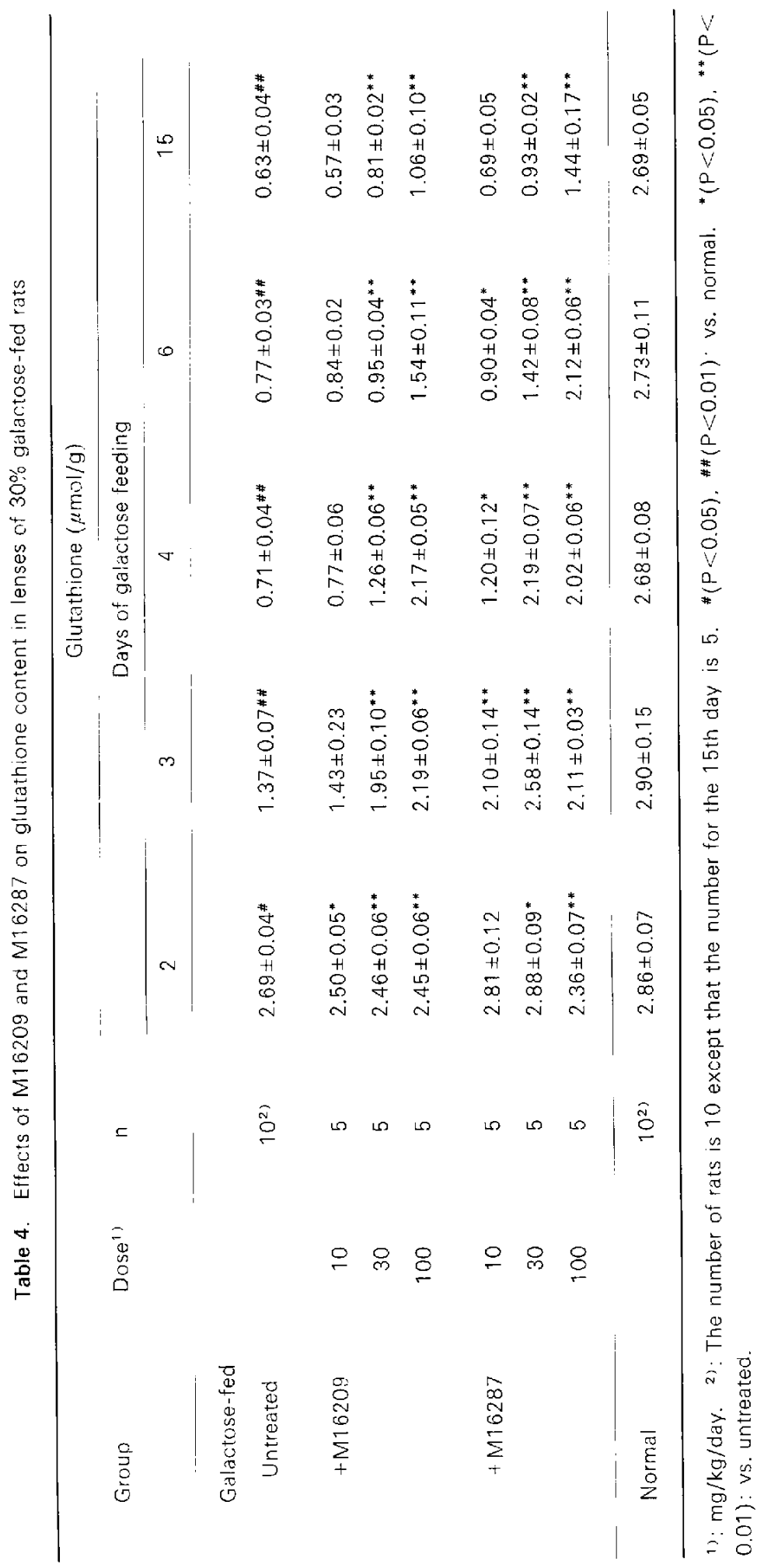


Table 5. Effects of M16209 and M16287 on NADPH and NADP+ contents in rat lenses removed on the 15 th day of $30 \%$ galactose feeding

\begin{tabular}{|c|c|c|c|c|}
\hline Group & Dose ${ }^{1)}$ & $\Pi$ & $\begin{array}{l}\text { NADPH } \\
(\mathrm{nmol} / \mathrm{g})\end{array}$ & $\begin{array}{c}\text { NADPt } \\
(\mathrm{nmol} / \mathrm{g})\end{array}$ \\
\hline \multicolumn{5}{|l|}{ Galactose-fed } \\
\hline Untreated & & 5 & $15.3 \pm 0.6^{\# \#}$ & $0.6 \pm 0.3^{\# \#}$ \\
\hline \multirow[t]{3}{*}{$-M 16209$} & 10 & 5 & $14.3 \pm 0.5$ & $1.7 \pm 0.1^{* *}$ \\
\hline & 30 & 5 & $14.7 \pm 0.5$ & $3.5 \pm 0.4^{* *}$ \\
\hline & 100 & 5 & $11.5 \pm 0.6^{* *}$ & $5.6 \pm 0.3^{* *}$ \\
\hline \multirow[t]{3}{*}{$+M 16287$} & 10 & 5 & $14.0 \pm 0.6$ & $3.0 \pm 0.4^{* *}$ \\
\hline & 30 & 5 & $12.9 \pm 0.7^{*}$ & $5.3 \pm 0.3^{* *}$ \\
\hline & 100 & 5 & $10.4 \pm 1.0^{* *}$ & $6.4 \pm 0.6^{* *}$ \\
\hline Normal & & 5 & $7.1 \pm 0.6$ & $8.3 \pm 0.4$ \\
\hline
\end{tabular}

1): $\mathrm{mg} / \mathrm{kg} / \mathrm{day} . \#(P<0.01)$ : vs. normal. * $(P<0.05),{ }^{* *}(P<0.01):$ vs. untreated.

Table 6. Effects of $\mathrm{M} 16209$ and $\mathrm{M} 16287$ on $\mathrm{Na}^{+}$and $\mathrm{K}^{+}$contents in rat lenses removed on the 15 th day of $30 \%$ galactose feeding

\begin{tabular}{|c|c|c|c|c|c|c|}
\hline Group & Dose ${ }^{11}$ & $n$ & $\begin{array}{c}\mathrm{Na}^{+} \\
(\mu \mathrm{mol} / \mathrm{g})\end{array}$ & $\begin{array}{c}\mathrm{K}^{+} \\
(\mu \mathrm{mol} / \mathrm{g})\end{array}$ & $\mathrm{Na}^{+} / \mathrm{K}^{+}$ & - \\
\hline $\begin{array}{c}\text { Galactose-fed } \\
\text { Untreated }\end{array}$ & & 5 & $29.8 \pm 0.8^{\# \#}$ & $50.3 \pm 1.9 \# \#$ & $0.60 \pm 0.03^{\# \#}$ & \\
\hline$+M 16209$ & $\begin{array}{r}10 \\
30 \\
100\end{array}$ & $\begin{array}{l}5 \\
5 \\
5\end{array}$ & $\begin{array}{l}26.1 \pm 1.4 \\
20.0 \pm 1.7^{* *} \\
19.3 \pm 1.4^{* *}\end{array}$ & $\begin{array}{l}46.3 \pm 0.8 \\
51.9 \pm 0.8 \\
52.3 \pm 1.2\end{array}$ & $\begin{array}{l}0.56 \pm 0.03 \\
0.39 \pm 0.03^{* *} \\
0.37 \pm 0.03^{* *}\end{array}$ & \\
\hline$+M 16287$ & $\begin{array}{r}10 \\
30 \\
100\end{array}$ & $\begin{array}{l}5 \\
5 \\
5\end{array}$ & $\begin{array}{l}26.0 \pm 0.9^{*} \\
18.2 \pm 1.5^{* *} \\
19.0 \pm 2.1^{* *}\end{array}$ & $\begin{array}{l}48.8 \pm 2.6 \\
49.2 \pm 1.8 \\
51.4 \pm 3.2\end{array}$ & $\begin{array}{l}0.54 \pm 0.04 \\
0.37 \pm 0.03^{* *} \\
0.37 \pm 0.02^{* *}\end{array}$ & \\
\hline Normal & & 5 & $19.0 \pm 2.5$ & $59.8 \pm 1.6$ & $0.32 \pm 0.04$ & \\
\hline
\end{tabular}

1): mg/kg/day. \#*(P<0.01): vs. normal. * $(P<0.05), * *(P<0.01)$ : vs. unteated.

feeding and reached the plateau before distinct clinical signs developed. Both $M$ 16209 and M16287 strongly suppressed the changes in these biochemical parameters at early stages, although the extent of suppression became smaller at late stages of galactose feeding. Especially, lens myo-inositol was undetectable in rats treated with those drugs at any doses after 2 weeks' galactose feeding. Nevertheless, treatment with M16209 or M16287 at 30 and $100 \mathrm{mg} / \mathrm{kg} /$ day almost completely suppressed cataract formation. These results support the suggestion of Kinoshita (2) that galactitol accumulation and GSH depletion are closely related to the pathogenesis of galactose cataract and aldose reductase inhibitors can prevent development of cataract by suppression of these abnormal metabolisms of galactitol and GSH. The data also suggested that myo-inositol, whose content decreased dramatically by galactose feeding, does not have a pivotal role in catarat pathogenesis.

The progression of lenticular changes leading to galactose cataract has been divided broadly into two different stages (15). The early stage is believed to be the initial vacuole formation due to osmotic swelling produced by the very rapid accumulation of galactitol levels of about $40-55 \mu \mathrm{mol} / \mathrm{g}$ lens $(16,17)$. On the other hand, the late stage is characterized by the breakdown of lenticular permeability. resulting in a fall in galactitol levels (17). These observations are consistent with our present results which showed that galactitol levels in the lenses of the rats in the 
untreated group rapidly increased and reached the plateau by the 4 th day of galactose feeding. and a galactitol level of more than 60 $\iota \mathrm{mol} / \mathrm{g}$ lens seemed to be prerequisite for vacuole formation.

Since aldose reductase demands NADPH as coenzyme, there is a possibility that NADPH decreases and NADP ${ }^{+}$increases concomitantly when the polyol pathway is activated. Contrary to the results of Lee et al. (18) who reported the drop of the ratio of

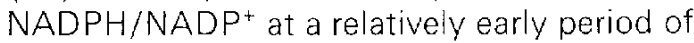
galactose feeding in rats, our results in the present study demonstrated the remarkable elevation of the ratio of $\mathrm{NADPH} / \mathrm{NADP}^{+}$ through the reciprocal changes in NADPH and NADP ${ }^{+}$content. This elevation of the ratio of NADPH/NADP ${ }^{+}$was also reported by Varma and Kinoshita (19), although only the $\mathrm{NADP}^{+}$content was altered by galactose feeding. The reason for these discrepancies is not clear, but our results may suggest that increased consumption of NADPH through the activated polyol pathway is compensated by increased production in the pentose phosphate pathway. It is also suggested that NADPH production in the pentose phosphate pathway is accelerated by increased demand for reduction of oxidized glutathione (20). Gonzalez et al. (21) have actually reported that the pentose phosphate pathway is activated in diabetes, and the activity of this pathway is suppressed by aldose reductase inhibitors. Then, the next questions may come up: Why does the GSH content decrease in spite of the increase in NADPH content? Why do aldose reductase inhibitors prevent the decrease in GSH content? Concerning the decrease in GSH content, Varma and Kinoshita (19) suggested a possibility of leakage of the molecule owing to increased membrane permeability. We found that galactitol accumulation was remarkable on the 2 nd day of galactose feeding. while the decrease in GSH content became obvious with one day's delay. This may indicate that galactitol accumulation impairs GSH metabolism and thus aldose reductase inhibitors can prevent $\mathrm{GSH}$ depletion. However, it remains to be clarified whether galactose feeding causes either an increase in GSH consumption, an impairment of $\mathrm{GSH}$ production, or both.
Another interesting question arises from the effects of our compounds on monovalent cation contents. The present study showed that our compounds did not restore the $\mathrm{K}^{+}$ content, although they effectively suppressed the increase in $\mathrm{Na}^{+}$. Abnormal ion content in sugar cataract has been explained by either increased membrane permeability (2) or decreased active transport dependent on $\mathrm{Na}^{+}$$\mathrm{K}^{+}$-ATPase activity (6). Our results can not be explained solely by either of them. since $\mathrm{Na}^{+}$ and $\mathrm{K}^{+}$should change reciprocally according to these theories. It seems possible to speculate that a $\mathrm{K}^{+}$-specific ion transport system independent of $\mathrm{Na}^{+}$may exist in the lens. If impairment of this $\mathrm{K}^{+}$-specific transport system is linked to the decrease in plasma membrane phosphoinositide, poor blockage of myo-inositol loss by our compounds is consistent with their ineffectiveness in restoring the $\mathrm{K}^{+}$content. Further investigation on this matter is required.

Finally, we wish to emphasize that M16209 and $M 16287$ are markedly effective for the prevention of galactose-induced cataract in rats and thus may be useful in treating some diabetic complications.

Acknowledgments: The authors wish to thank Miss Tomoko Hatsuya, Miss Akemi Kurita and Miss Michiko Machida for excellent technical assistance.

\section{References}

1 van Heyningen, R.: Formation of polyols by the lens of the rat with sugar cataract. Nature 184 , 194-195 (1959)

2 Kinoshita, J.H.: Cataracts in galactosemia. Invest. Ophthalmol. 4, 786-799 (1965)

3 Kinoshita, J.H., Kador, P.F., Robison, W.G., Datilis, M.B., Cobo, L.M. and Kupter, C.: Aldose reductase and complications of diabetes. Ann. Intern. Med. 101, 82-91 (1984)

4 Raskin, P. and Rosenstock, J.: Aldose reductase inhibitors and diabetic complications. Am. J. Med. 83, 298-306 (1987)

5 Greene, D.A., Lewis, R.A., Lattimer, S.A. and Brown, M.J.: Selective effects of myo-inositol administration on sciatic and tibial motor nerve conduction parameters in the streptozotocindiabetic rat. Diabetes 31, 573-578 (1982)

6 Greene, D.A. and Lattimer, S.A.: Action of sorbinil in diabetic peripheral nerve: Relationship of polyol (sorbitol) pathway inhibition to a myoinositol-mediated defect in sodium-potassium 
ATPase activity. Diabetes 33, 712-716 (1984)

7 Ohrloff, C., Hockwin, O., Olson R. and Dickman, S.: Glutathione peroxidase, glutathione reductase and superoxide dismutase in the aging lens. Current Eye Res. 3, 109-115 (1984)

8 Gonzalez, A.M., Sochor, M. and McLean, P.: The effect of an aidose reductase inhibitor (Sorbinil) on the level of metabolites in lenses of diabetic rats. Diabetes 32, 482-485 (1983)

9 Inagaki, K., Miwa, I., Yashiro, T. and Okuda, J.: Inhibition of aldose reductases from rat and bovine lenses by hydantoin derivatives. Chem. Pharm. Bull. (Tokyo) 30, 3244-3254 (1982)

10 Sippel, T.O.: Changes in the water, protein and glutathione contents of the lens in the course of galactose cataract development in rats. invest. Ophthalmol. 5, 568-575 (1966)

11 Miwa, I. Kanbara, M., Wakazono, H. and Okuda, J.: Analysis of sorbitol, galactitol and myoinositol in lens and sciatic nerve by high-performance liquid chromatography. Anal. Biochem. 173, 39-44 (1988)

12 Hissin, P.J. and Hilf, R.: A fluorometric method for determination of oxidized and reduced glutathione in tissues. Anal. Biochem. 74, 214226 (1976)

13 Passonneau, J.V. and Lowry, O.H.: Measurement by enzymatic cycling. In Methods of Enzymatic Analysis, Edited by Bergmeyer, H.U., Vol. 4, p. 2059-2072. Academic Press. Inc. New York (1974)

14 Kuriyama, H., Sasaki, K. and Fukuda, M.: Studies on diabetic cataract in rats induced by streptozotocin. II. Biochemical examinations of rat lenses in relation to cataract stages. Ophthalmic Res. 15, 191-197(1983)

15 Kinoshita, J.H. and Merola, L.O.: Hydration of the lens during the development of galactose cataract. Invest. Ophthalmol. 3,577-584 (1964)

16 Simard-Duquesne, N., Greseiin, E., Gonzalez, R. and Dvornik, D.: Prevention of cataract development in severely galactosemic rats by the aldose reductase inhibitor, tolrestat. Proc. Soc. Exp. Biol. Med. 178, 599-605 (1985)

17 Simard-Duquesne, N. and Dvornik, D.: Galactitol accumulation and irreversible lens opacities in galactosemic rats. invest. Ophthalmol. $12,82-83$ (1973)

18 Lee, S.-M., Schade, S.Z. and Doughty, C.C.: Aldose reductase, NADPH and NADP+ in normal, galactose-fed and diabetic rat lens. Biochim. Biophys. Acta 841, 247-253 (1985)

19 Varma, S.D. and Kinoshita, J.H.: Sorbitol pathway in diabetic and galactosemic rat lens. Blochim. Biophys. Acta 338, 632-640 (1974)

20 Giblin, F.J., Nies, D.E. and Reddy, V.N.: Stimulation of the hexose monophosphate shunt in rabbit lens in response to the oxidation of glutathione. Exp. Eye Res. 33, 289-298 (1981)

21 Gonzalez, A.M., Sochor, M. Hothersall, J.S. and McLean, P.: Effect of aldose reductase inhibitor (Sorbinil) on integration of polyol pathway, pentose phosphate pathway. and glycolytic route in diabetic rat lens. Diabetes $35,1200-1205$ (1986) 